\title{
Bound States for Pseudoharmonic Potential of the Dirac Equation with Spin and Pseudo-Spin Symmetry via Laplace Transform Approach
}

\author{
B. BisWAS ${ }^{a, *}$ AND S. DeBnATH ${ }^{b}$ \\ ${ }^{a}$ Department of Mathematics, P.K.H.N. Mahavidyalaya, Howrah, India \\ ${ }^{b}$ Department of Mathematics, Jadavpur University, Kolkata 700 032, India
}

(Received May 7, 2016; in final form August 3, 2016)

\begin{abstract}
Bound state solutions of the Dirac equation for the pseudoharmonic potential with spin and pseudo-spin symmetry are studied in this paper. To obtain the exactly normalized bound state wave function and energy expressions we have used the Laplace transform approach.
\end{abstract}

DOI: 10.12693/APhysPolA.130.692

PACS/topics: 03.65.-w, 03.65.Ge, 03.65.Pm

\section{Introduction}

In the field of relativistic quantum mechanics, the Dirac equation plays an important role for spin- $1 / 2$ particles. This equation has been used extensively to study the relativistic heavy ion collisions, heavy ion spectroscopy and more recently in laser-matter interaction [1] and condensed matter physics [2].

The most interesting feature for the Dirac equation is the concept of spin and pseudospin symmetries. Although these symmetries of the Dirac Hamiltonian were discovered long ago, there has been a renewed interest in obtaining the solutions of the Dirac equations for some typical potentials under spin symmetry and pseudo-spin symmetry cases. The idea about spin symmetry and pseudo-spin symmetry with the nuclear shell model has been introduced in [3]. This idea has been widely used in explaining a number of phenomena in nuclear physics and related areas. Spin and pseudo-spin symmetric concepts have been used in the studies of certain aspects of deformed and exotic nuclei.

Spin symmetry is relevant to meson with one heavy quark, which is being used to explain the absence of quark spin-orbit splitting (spin doublets) observed in heavy-light quark mesons [4] and pseudo-spin symmetry concept has been successfully used to explain different phenomena in nuclear structure including deformation, superdeformation, identical bands, exotic nuclei, and degeneracies of some shell model orbitals in nuclei (pseudospin doublets) [5,6]. In recent times, many works have been done to solve the Dirac equation to obtain the bound states energy spectra and the corresponding eigenfunctions. Ginocchio [7-12] deduced that a Dirac Hamiltonian with scalar $S(r)$ and vector $V(r)$ harmonic oscillator potentials when $V(r)=S(r)$ possesses a spin symmetry as well as a U(3) symmetry, whereas a Dirac

\footnotetext{
*corresponding author; e-mail: biswasdebnathju@gmail.com
}

Hamiltonian for the case of $V(r)+S(r)=0$ or $V(r)=$ $-S(r)$ possesses a pseudo-spin symmetry and a pseudo$\mathrm{U}(3)$ symmetry. As introduced in nuclear theory, the pseudo-spin symmetry refers to a quasi-degeneracy of the single-nucleon doublets which can be characterized with the non-relativistic quantum mechanics $\left(n, l, j=l+\frac{1}{2}\right)$ and $\left(n-1, l+2, j=l+\frac{3}{2}\right)$, where $n, l$ and $j$ are the single-nucleon radial, orbital and total angular momentum quantum numbers for a single particle, respectively. The total angular momentum is given as $j=\bar{l}+\bar{s}$, where $\bar{l}=l+1$ is a pseudo-angular momentum and $\bar{s}=\frac{1}{2}$ is a pseudo-spin angular momentum. The orbital and pseudo-orbital angular momentum quantum numbers for spin symmetry $l$ and pseudo-spin symmetry $\bar{l}$ refer to the upper- and lower-spinor components: $F_{n, k}(r)$ and $G_{n, k}(r)$, respectively.

The spin symmetry occurs when the difference between the repulsive Lorentz vector potential $V(r)$ and attractive Lorentz scalar potential $S(r)$ in the Dirac Hamiltonian is a constant, that is, $\Delta(r)=V(r)-S(r)=$ const and pseudo-spin symmetry occurs when the sum of two potential is a constant, that is, $\Sigma(r)=V(r)+S(r)=$ const.

Recently, many researchers have applied spin and pseudo-spin symmetry conditions on a number of potentials. These potentials include: the Hulthén potential [13], the Eckart potential [14], the Pöschl-Teller potential [15, 16], the Rosen-Morse potential [17], harmonic potential [18], the Manning-Rosen potential [19], the Wood-Saxon potential [20], the Kratzer potential with angle dependent potential [21], the Scarf potential [22], the Hua potential [23]. In this study we consider the pseudoharmonic potential [24-26] given as

$$
V(r)=D_{0}\left(\frac{r}{r_{0}}-\frac{r_{0}}{r}\right)^{2}
$$

where $r_{0}$ is an equilibrium distance and $D_{0}$ is a constant related to the dissociation energy of a molecule.

There are various methods to solve spin and pseudo-spin symmetry problems including the centrifu- 
gal approximation ranging from the asymptotic iteration method (AIM) [14, 27], the Nikiforov-Uvarov method $(\mathrm{N}-\mathrm{U})$, supersymmetric and shape invariance method [28]. Recently, solutions of the Schrödinger and Klein-Gordon $(\mathrm{K}-\mathrm{G})$ equations with several potentials have been investigated using various methods [29-43]. Here we adopt the Laplace transform approach (LTA) to cultivate the bound state energy eigenvalues and the corresponding eigenfunctions for pseudoharmonic potential with the repulsive Lorentz vector potential $V(r)$ and attractive Lorentz scalar potential $S(r)$ for the Dirac equation. In order to obtain the relativistic bound state energy eigenvalues and the corresponding Dirac spinors, we use a different and very economical method, called the Laplace transform approach within the framework of the pseudo-spin and the spin symmetry concept. The LTA is an integral transform which has been used by many authors [44, 45].

This work is organized as follows: To make it selfcontained, we present Laplace transform approach with necessary formulae to perform our calculations in the next section. In Sect. 3, we have formulated the Dirac equation to Schrödinger-like equation for suitable application of LTA. In Sects. 4 and 5, we consider the spin symmetric and the pseudo-spin symmetric solutions of the pseudoharmonic potential for any $k$ state, respectively. The last section is kept for conclusive remark.

\section{Laplace transform approach}

Suppose the differential equation contain a term of the form $t^{m} y^{(n)}(t)$ i.e., $t^{m} \frac{\mathrm{d}^{n} y(t)}{\mathrm{d} t^{n}}$. Then the Laplace transform of the term is represented by

$$
L\left(t^{m} \frac{\mathrm{d}^{n} y(t)}{\mathrm{d} t^{n}}\right)=(-1)^{m} \frac{\mathrm{d}^{m}}{\mathrm{~d} s^{m}} L\left(y^{(n)}(t)\right) .
$$

So,

$$
L\left[t y^{\prime \prime}(t)\right]=(-1) \frac{\mathrm{d}}{\mathrm{d} s} L\left[y^{\prime \prime}(t)\right]
$$

Again, another important theorem for Laplace transform of first order and second order derivative for continuous $y(t)$ and $y^{\prime}(t)$ with $t \geq 0$ of exponential order $\sigma$ as $t \rightarrow \infty$ and if $y^{\prime}(t)$ and $y^{\prime \prime}(t)$ is of class A, then the Laplace transforms of $y^{\prime}(t)$ and $y^{\prime \prime}(t)$ for $s>\sigma$ are given by

$$
L\left[y^{\prime}(t)\right]=s L[y(t)]-y(0)
$$

and

$$
L\left[y^{\prime \prime}(t)\right]=s^{2} L[y(t)]-s y(0)-y^{\prime}(0) .
$$

One of the most important formula used in our calculation is: if $y(t)$ is a function of class $\mathrm{A}$, then

$$
L\left[t^{n} y(t)\right]=(-1)^{n} \frac{\mathrm{d}^{n} f(s)}{\mathrm{d} s^{n}},
$$

where $f(s)=L[y(t)]=\int_{0}^{\infty} y(t) \mathrm{e}^{-t s} \mathrm{~d} t$ and $n=1,2,3, \ldots$

After conversion of second order differential equation to a first order one, we further apply the inverse Laplace transform to obtain the wave function. The relevant formulae for inverse Laplace transform are followed from Ref. [46].

\section{Transformation of Dirac equation to Schródinger-like equation}

The Dirac equation of a nucleon with mass $M$ moving in an attractive scalar potential $S(r)$ and a repulsive vector potential $V(r)$ for spin- $\frac{1}{2}$ particles in the relativistic unit $(\hbar=c=1)$ is [47]:

$$
[\alpha p+\beta(M+S(r))] \psi(r)=[E-V(r)] \psi(r),
$$

where $E$ is the relativistic energy of the system, $p=-\mathrm{i} \nabla$ is the three-dimensional momentum operator and $M$ is the mass of the fermionic particle. $\alpha, \beta$ are the $4 \times 4$ Dirac matrices given as [47]:

$$
\alpha=\left(\begin{array}{cc}
0 & \sigma_{i} \\
\sigma_{i} & 0
\end{array}\right), \quad \beta=\left(\begin{array}{cc}
I & 0 \\
0 & -I
\end{array}\right),
$$

where $I$ is a $2 \times 2$ unit matrix and $\sigma_{i}$ are the Pauli threevector matrices

$$
\sigma_{1}=\left(\begin{array}{ll}
0 & 1 \\
1 & 0
\end{array}\right), \sigma_{2}=\left(\begin{array}{cc}
0 & -\mathrm{i} \\
\mathrm{i} & 0
\end{array}\right), \sigma_{3}=\left(\begin{array}{cc}
1 & 0 \\
0 & -1
\end{array}\right)
$$

The eigenvalues of the spin-orbit coupling operator are $k=\left(j+\frac{1}{2}\right)>0, k=-\left(j+\frac{1}{2}\right)<0$ for the unaligned spin $j=l-\frac{1}{2}$ and aligned spin $j=l+\frac{1}{2}$, respectively. The set $\left(H, K, J^{2}, J_{z}\right)$ forms a complete set of conserved quantities. Thus, we can write the spinors as [47]:

$$
\psi_{n k}(r)=\frac{1}{r}\left(\begin{array}{cc}
F_{n k}(r) & Y_{j m}^{l}(\theta, \phi) \\
\mathrm{i} G_{n k}(r) & Y_{j m}^{\bar{l}}(\theta, \phi)
\end{array}\right),
$$

where $F_{n k}(r), G_{n k}(r)$ represent the upper and lower components of the Dirac spinors and $\bar{l}$ is pseudo-orbital angular momentum, which is defined as $\bar{l}=l+1$ for the aligned spin $j=\bar{l}-\frac{1}{2}$ and $\bar{l}=l-1$ for the unaligned spin $j=\boldsymbol{l}+\frac{1}{2} \cdot Y_{j m}^{l}(\theta, \phi), Y_{j m}^{\bar{l}}(\theta, \phi)$ are the spin and pseudo-spin spherical harmonics and $m$ is the projection on the $z$-axis. Using well-known identities,

$$
\begin{gathered}
(\sigma \cdot A)(\sigma \cdot B)=A \cdot B+\mathrm{i} \sigma \cdot(A \times B), \\
\sigma \cdot p=\sigma \cdot \hat{r}\left(\hat{r} \cdot p+\mathrm{i} \frac{\sigma \cdot L}{r}\right),
\end{gathered}
$$

as well as the relations

$$
\begin{aligned}
& (\sigma \cdot L) Y_{j m}^{\bar{l}}(\theta, \phi)=(k-1) Y_{j m}^{\bar{l}}(\theta, \phi), \\
& (\sigma \cdot L) Y_{j m}^{l}(\theta, \phi)=-(k+1) Y_{j m}^{l}(\theta, \phi), \\
& (\sigma \cdot \hat{r}) Y_{j m}^{l}(\theta, \phi)=-Y_{j m}^{\bar{l}}(\theta, \phi), \\
& (\sigma \cdot \hat{r}) Y_{j m}^{\bar{l}}(\theta, \phi)=-Y_{j m}^{l}(\theta, \phi) .
\end{aligned}
$$

The coupled first-order Dirac equations are

$$
\begin{aligned}
& \left(\frac{\mathrm{d}}{\mathrm{d} r}+\frac{k}{r}\right) F_{n k}(r)=\left[M+E_{n k}-\Delta(r)\right] G_{n k}(r), \\
& \left(\frac{\mathrm{d}}{\mathrm{d} r}-\frac{k}{r}\right) G_{n k}(r)=\left[M-E_{n k}+\Sigma(r)\right] F_{n k}(r),
\end{aligned}
$$

where

$$
\begin{aligned}
& \Delta(r)=V(r)-S(r), \\
& \Sigma(r)=V(r)+S(r) .
\end{aligned}
$$

Eliminating $F_{n k}(r)$ and $G_{n k}(r)$ in Eqs. (13) and (14), we obtain the second-order Schrödinger-like equation 


$$
\begin{gathered}
\quad\left\{\frac{\mathrm{d}^{2}}{\mathrm{~d} r^{2}}-\frac{k(k+1)}{r^{2}}-\left[M+E_{n k}-\Delta(r)\right]\right. \\
\left.\times\left[M-E_{n k}+\Sigma(r)\right]+\frac{\frac{\mathrm{d} \Delta(r)}{\mathrm{d} r}\left(\frac{\mathrm{d}}{\mathrm{d} r}+\frac{k}{r}\right)}{M+E_{n k}-\Delta(r)}\right\} F_{n k}(r)=0, \\
\quad\left\{\frac{\mathrm{d}^{2}}{\mathrm{~d} r^{2}}-\frac{k(k-1)}{r^{2}}-\left[M+E_{n k}-\Delta(r)\right]\right. \\
\left.\times\left[M-E_{n k}+\Sigma(r)\right]+\frac{\frac{\mathrm{d} \Sigma(r)}{\mathrm{d} r}\left(\frac{\mathrm{d}}{\mathrm{d} r}-\frac{k}{r}\right)}{M-E_{n k}-\Sigma(r)}\right\} G_{n k}(r)=0,
\end{gathered}
$$

where $k(k-1)=\bar{l}(\bar{l}+1)$ and $k(k+1)=l(l+1)$.

We consider bound state solutions that demand the radial components satisfying $F_{n k}(0)=G_{n k}(0)=0$ and $F_{n k}(\infty)=G_{n k}(\infty)=0$.

\section{Bound state solutions of the pseudoharmonic potential for the spin symmetric case}

In the case of exact spin symmetry $\frac{\mathrm{d} \Delta(r)}{\mathrm{d} r}=0$, i.e., $\Delta(r)=C=$ const, Eq. (17) becomes

$$
\begin{aligned}
& \left\{\frac{\mathrm{d}^{2}}{\mathrm{~d} r^{2}}-\frac{k(k+1)}{r^{2}}-\left[M+E_{n k}-\Delta(r)\right]\right. \\
& \left.\times\left[M-E_{n k}+\Sigma(r)\right]\right\} F_{n k}(r)=0,
\end{aligned}
$$

where $k=l$ for $k<0$ and $k=-(l+1)$ for $k>0$. The energy eigenvalues depend on $n$ and $l$, i.e., $E_{n k}=$ $E(n, l(l+1))$, which is well known as the exact spin symmetry. We assume that $\sum(r)$ is the pseudoharmonic potential and Eq. (19) takes the form with this potential as

$$
\begin{aligned}
& \left\{\frac{\mathrm{d}^{2}}{\mathrm{~d} r^{2}}-\frac{k(k+1)}{r^{2}}-\left(M+E_{n k}-C\right)\left[M-E_{n k}\right.\right. \\
& \left.\left.+D_{0}\left(\frac{r}{r_{0}}-\frac{r_{0}}{r}\right)^{2}\right]\right\} F_{n k}(r)=0 .
\end{aligned}
$$

Now defining the new variable $x=r^{2}$ and the function $F_{n k}(r)=x^{-\frac{\nu}{2}} \varphi(x)$, we get

$$
\left[x \frac{\mathrm{d}^{2}}{\mathrm{~d} x^{2}}-\left(\nu-\frac{1}{2}\right) \frac{\mathrm{d}}{\mathrm{d} x}-\frac{1}{4}\left(\mu^{2} x-\varepsilon^{2}\right)\right] \varphi(x)=0,
$$

where

$$
\begin{aligned}
& \nu(\nu+1)=k(k+1)+\left(M+E_{n k}-C\right) D_{0} r_{0}^{2}, \\
& \mu^{2}=\frac{D_{0}}{r_{0}^{2}}\left(M+E_{n k}-C\right), \\
& \varepsilon^{2}=\left(2 D_{0}+E_{n k}-M\right)\left(M+E_{n k}-C\right) .
\end{aligned}
$$

This form of equation is suitable for the application of LTA which is described above in Sect. 2 and applying the LTA to the above equation we obtain a first order differential equation

$$
\left(t^{2}-\frac{\mu^{2}}{4}\right) \frac{\mathrm{d} f(t)}{\mathrm{d} t}+\left[\left(\nu+\frac{3}{2}\right) t-\frac{\varepsilon^{2}}{4}\right] f(t)=0,
$$

where $f(t)=L[\varphi(x)]$ and the solution is given by

$$
f(t)=N\left(t+\frac{\mu}{2}\right)^{-\left(\nu+\frac{3}{2}\right)}\left(\frac{t-\frac{\mu}{2}}{t+\frac{\mu}{2}}\right)^{\frac{\varepsilon^{2}}{4 \mu}-\frac{1}{2}\left(\nu+\frac{3}{2}\right)} .
$$

The wave functions required to be single-valued but the term $\left(\frac{t-\frac{\mu}{2}}{t+\frac{\varepsilon^{2}}{2}}\right)^{\frac{\varepsilon^{2}}{4 \mu}-\frac{1}{2}\left(\nu+\frac{3}{2}\right)}$ is multi-valued. Therefore, we must have to take

$$
\frac{\varepsilon^{2}}{4 \mu}-\frac{1}{2}\left(\nu+\frac{3}{2}\right)=n \quad(n=0,1,2, \ldots) .
$$

Now applying a simple series expansion to Eq. (24) we obtain

$$
f(t)=N^{\prime} \sum_{m=0}^{n} \frac{(-1)^{m} n !}{(n-m) ! m !}\left(t+\frac{\mu}{2}\right)^{-\nu+\frac{3}{2}-m},
$$

where $N^{\prime}$ is a constant. Using the inverse Laplace transformation in Eq. (26), we get that

$$
\begin{gathered}
\varphi(x)=N^{\prime \prime} x^{\nu+\frac{1}{2}} \mathrm{e}^{-\frac{\mu x}{2}} \sum_{m=0}^{n} \frac{(-1)^{m} n !}{(n-m) ! m !} \frac{\Gamma\left(\nu+\frac{1}{2}\right)}{\Gamma\left(\nu+\frac{3}{2}+m\right)} x^{m}= \\
N^{\prime \prime} x^{\nu+\frac{1}{2}} \mathrm{e}^{-\frac{\mu x}{2}}{ }_{1} F_{1}\left(-n ; \nu+\frac{3}{2} ; x\right),
\end{gathered}
$$

where ${ }_{1} F_{1}\left(-n ; \nu+\frac{3}{2} ; x\right)$ is the notation of confluent hypergeometric function [48].

Finally, we obtain the upper component of the Dirac spinor as

$$
F_{n k}(r)=N r^{\nu+1} \mathrm{e}^{-\frac{\mu r^{2}}{2}}{ }_{1} F_{1}\left(-n ; \nu+\frac{3}{2} ; r^{2}\right),
$$

where $N$ is the normalization constant.

In order to find the lower component spinor, the recurrence relation of the confluent hypergeometric function

$$
\frac{\mathrm{d}}{\mathrm{d} r}{ }_{1} F_{1}(a ; b ; r)=\frac{a}{b}{ }_{1} F_{1}(a+1 ; b+1 ; r)
$$

is used to evaluate Eq. (13) and this is obtained for spin symmetry case (i.e. for $\Delta(r)=C=$ const) as

$$
\begin{aligned}
& G_{n k}(r)=\frac{N r^{\nu+2} \mathrm{e}^{\frac{\mu r^{2}}{2}}}{M+E_{n k}-C} \\
& \quad \times\left\{\frac{-2 n}{\nu+\frac{3}{2}}{ }_{1} F_{1}\left(-n+1 ; \nu+\frac{5}{2} ; r^{2}\right)\right. \\
& \left.\quad+\left(\frac{k+\nu+1}{r^{2}}-\mu\right){ }_{1} F_{1}\left(-n ; \nu+\frac{3}{2} ; r^{2}\right)\right\} .
\end{aligned}
$$

Using Eqs. (22) and (25), an explicit expression for the energy eigenvalues of the Dirac equation with the pseudoharmonic potential under the spin symmetry condition is obtained as

$$
\begin{aligned}
& \frac{r_{0}}{\sqrt{D_{0}}}\left(2 D_{0}+E-M\right) \sqrt{M+E-C} \\
- & \sqrt{(2 k+1)^{2}+4 D_{0} r_{0}^{2}(M+E-C)}=4\left(n+\frac{1}{2}\right) .
\end{aligned}
$$

According to Ginocchio [12] there are only positive energy eigenvalues and no bound state negative energy eigenvalues exist in the spin limit. Therefore, in the spin limit, only positive energy eigenvalues are chosen. 


\section{Bound state solutions of the pseudoharmonic potential for the pseudo-spin symmetric case}

In the case of exact pseudo-spin symmetry $\frac{\mathrm{d} \sum(r)}{\mathrm{d} r}=0$, i.e., $\sum(r)=C_{p s}=$ const, Eq. (18) becomes

$$
\begin{gathered}
\left\{\frac{\mathrm{d}^{2}}{\mathrm{~d} r^{2}}-\frac{k(k-1)}{r^{2}}-\left(M+E_{n k}-\Delta(r)\right)\right. \\
\left.\times\left(M-E_{n k}+C_{p s}\right)\right\} G_{n k}(r)=0,
\end{gathered}
$$

where $k$ is related to the pseudo-orbital angular quantum number $\bar{l}$ as $k(k-1)=\bar{l}(\bar{l}+1), k=-\bar{l}$ for $k<0$ and $k=(\bar{l}+1)$ for $k>0$, which implies that $j=\bar{l} \pm \frac{1}{2}$ are degenerate for $\bar{l} \neq 0$. It is required that the upper and lower spinor components must satisfy the following boundary conditions: $F_{n k}(0)=G_{n k}(0)=0$ and $F_{n k}(\infty)=G_{n k}(\infty)=0$ for bound state solutions. The energy eigenvalues depend on $n$ and $\bar{l}$, i.e., $E_{n k}=E(n, \bar{l}(\bar{l}+1))$, which is well known as the exact pseudo-spin symmetry. We assume that $\Sigma(r)$ is the pseudoharmonic potential and Eq. (32) takes the form with this potential as

$$
\begin{aligned}
& \left\{\frac{\mathrm{d}^{2}}{\mathrm{~d} r^{2}}-\frac{\bar{l}(\bar{l}+1)}{r^{2}}-\left(M+E_{n k}-D_{0}\left(\frac{r}{r_{0}}-\frac{r_{0}}{r}\right)^{2}\right)\right. \\
& \left.\times\left(M-E_{n k}+C_{p s}\right)\right\} G_{n k}(r)=0 .
\end{aligned}
$$

Now applying same procedure as above by defining the new variable $x=r^{2}$ and the function $G_{n k}(r)=x^{-\frac{\nu}{2}} \varphi(x)$, we get

$$
\left[x \frac{\mathrm{d}^{2}}{\mathrm{~d} x^{2}}-\left(\nu-\frac{1}{2}\right) \frac{\mathrm{d}}{\mathrm{d} x}-\frac{1}{4}\left(\mu^{2} x-\varepsilon^{2}\right)\right] \varphi(x)=0,
$$

where

$$
\begin{aligned}
& \nu(\nu+1)=k(k-1)-\left(M-E_{n k}+C_{p s}\right) D_{0} r_{0}^{2}, \\
& \mu^{2}=-\frac{D_{0}}{r_{0}^{2}}\left(M-E_{n k}+C_{p s}\right), \\
& \varepsilon^{2}=-\left(2 D_{0}+E_{n k}+M\right)\left(M-E_{n k}+C_{p s}\right) .
\end{aligned}
$$

Equation (37) is similar to Eq. (21) and so, via the calculations like the above one, the lower component of the Dirac spinor can be obtained as

$$
G_{n k}(r)=\tilde{N} r^{\nu+1} \mathrm{e}^{-\frac{\mu r^{2}}{2}}{ }_{1} F_{1}\left(-n ; \nu+\frac{3}{2} ; r^{2}\right),
$$

where $\tilde{N}$ is the normalization constant and ${ }_{1} F_{1}(-n ; \nu+$ $\left.\frac{3}{2} ; r^{2}\right)$ is confluent hypergeometric function.

In order to find the upper component spinor, the recurrence relation of the confluent hypergeometric function

$$
\frac{\mathrm{d}}{\mathrm{d} r}{ }_{1} F_{1}(a ; b ; r)=\frac{a}{b}{ }_{1} F_{1}(a+1 ; b+1 ; r)
$$

is used to evaluate Eq. (14) and this is obtained for pseudo-spin symmetry case (i.e. for $\Sigma(r)=C_{p s}=$ const) as

$$
F_{n k}(r)=\frac{N r^{\nu+2} \mathrm{e}^{\frac{\mu r^{2}}{2}}}{M-E_{n k}+C_{p s}}\left[\frac{-2 n}{\nu+\frac{3}{2}}{ }_{1} F_{1}\left(-n+1 ; \nu+\frac{5}{2} ; r^{2}\right)\right.
$$

$$
\left.+\left(\frac{\nu+1-k}{r^{2}}-\mu\right){ }_{1} F_{1}\left(-n ; \nu+\frac{3}{2} ; r^{2}\right)\right],
$$

where $\tilde{N}$ is the normalization constant.

Also, in the similar fashion as obtained in the case of the spin symmetry condition, an explicit expression for the energy eigenvalues of the Dirac equation with the pseudoharmonic potential under the pseudospin symmetry is obtained as

$$
\begin{aligned}
& \frac{r_{0}}{\sqrt{D_{0}}}\left(2 D_{0}+E+M\right) \sqrt{E-M-C} \\
-\sqrt{(2 k-1)^{2}-4 D_{0} r_{0}^{2}(M-E+C)} & =4\left(n+\frac{1}{2}\right),
\end{aligned}
$$

where $k(k-1)=\bar{l}(\bar{l}+1)$. It has been shown that there are only negative energy eigenvalues and no bound positive energy eigenvalues exist in the pseudo-spin limit [12]. Therefore, in the pseudo-spin limit, only negative energy eigenvalues are chosen.

\section{Conclusions}

In this work, we have obtained the bound state solutions of the Dirac equation with spin and pseudo-spin symmetry for scalar and vector pseudoharmonic potential of the form $V(r)=D_{0}\left(\frac{r}{r_{0}}-\frac{r_{0}}{r}\right)^{2}$ depending on the spatial coordinate $r$. The variation of the above potential according to coordinate $r$ is given in Fig. 1. The twocomponent spinors and the corresponding energy equation have been obtained within the framework of the LTA which is a powerful algebraic treatment for solving the second-order differential equation via conversion of it into a more simpler form. The upper and lower component spinors have been expressed in terms of the confluent hypergeometric functions.

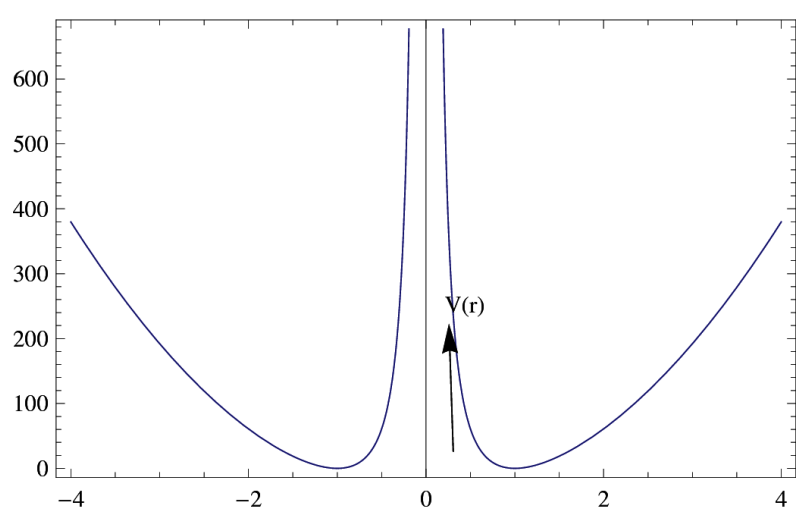

Fig. 1. Graphical representation of the pseudoharmonic potential for $D_{0}=27$ and $r_{0}=1$ for different values of $r$.

\section{References}

[1] Y.L. Salamin, S. Hu, K.Z. Hatsagortsyan, C.H. Keitel, Phys. Rep. 427, 41 (2006).

[2] M.I. Katsnelson, K.S. Novoselov, A.K. Geim, Nature Phys. 2, 620 (2006) 
[3] K.T. Hecht, A. Adler, Nucl. Phys. A 137, 129 (1969).

[4] P.R. Page, T. Goldman, J.N. Ginocchio, Phys. Rev. Lett. 86, 204 (2001).

[5] J. Dudek, W. Nazarewich, Z. Szymanski, G.A. Leander, Phys. Rev. Lett. 59, 1405 (1987).

[6] A. Bohr, I. Hamamoto, B.R. Mottelson, Phys. Scr. 26, 267 (1982)

[7] J.N. Ginocchio, Phys. Rev. Lett. 78, 436 (1997).

[8] J.N. Ginocchio, D.G. Madland, Phys. Rev. C 57, 1167 (1998)

[9] J.N. Ginocchio, Phys. Rep. 315, 231 (1999).

[10] J.N. Ginocchio, Phys. Rev. C 69, 034318 (2004).

[11] J.N. Ginocchio, Phys. Rep. 414, 165 (2005).

[12] J.N. Ginocchio, Phys. Rev. Lett. 95, 252501 (2005).

[13] A. Soylu, O. Bayrak, I. Boztosun, J. Math. Phys. 48, 082302 (2007).

[14] L.H. Zhang, X.P. Li, C.S. Jia, Phys. Lett. A $\mathbf{3 7 2}$, 2201 (2008)

[15] C.S. Jia, P. Gao, Y.F. Diao, L.Z. Yi, X.J. Xie, Eur. Phys. J. A 34, 41 (2007).

[16] D. Agboola, Pramana J. Phys. 76, 875 (2011).

[17] S.M. Ikhdair, J. Math. Phys. 51, 023525 (2010).

[18] J.Y. Guo, X.Z. Fang, F.X. Xu, Nucl. Phys. A 757, $411(2005)$.

[19] T. Chen, J.Y. Liu, C.S. Jia, Phys. Scr. 79, 055002 (2009).

[20] J.Y. Guo, Z.Q. Sheng, Phys. Lett. A 338, 90 (2005).

[21] C. Berkdermir, R. Sever, J. Phys. A Math. Theor. 41, 0453302 (2009).

[22] B.J. Falayel, K.J. Oyewumi, Afr. Rev. Phys. 6, 0025 (2011).

[23] P. Boonserm, M. Visser, arXiv:1005.4483.

[24] S. Erkoc, R. Sever, Phys. Rev. A 37, 2687 (1988).

[25] I.I. Goldman, V.D. Krivchenkov, Problems in Quantum Mechanics, Pergamon Press, New York 1961.

[26] Y. Weissman, J. Jortner, Phys. Lett. A 70, 177 (1979).

[27] A.N. Ikot, E. Maghsoodi, S. Zarrinkamar, H. Hassanabadi, Few-Body Syst. 54, 2027 (2013).
[28] R. Dutt, A. Gangopadhyaya, U.P. Sukhatme, Am. J. Phys. 65, 400 (1997).

[29] S.M. Ikhdair, R. Sever, J. Mol. Struct. Theochem. 806, 155 (2007).

[30] S. Debnath, B. Biswas, EJTP 9, 191 (2012).

[31] B. Biswas, S. Debnath, Bull. Cal. Math. Soc. 104, 481 (2012).

[32] B. Biswas, S. Debnath, Afr. Rev. Phys. 8, 0018 (2013).

[33] B. Biswas, S. Debnath, Afr. Rev. Phys. 8, 0030 (2013).

[34] S. Meyur, S. Debnath, Bul. J. Phys. 35, 22 (2008).

[35] S. Meyur, S. Debnath, Bul. J. Phys. 37, 1697 (2006).

[36] S. Meyur, S. Debnath, Mod. Phys. Lett. A 23, 2077 (2008).

[37] S. Meyur, S. Debnath, Ukr. J. Phys. 53, 713 (2008).

[38] S. Meyur, S. Debnath, Lat. Am. J. Phys. Educ. 3, 300 (2009).

[39] S.M. Ikhdair, R. Sever, Cent. Eur. J. Phys. 5, 516 (2007).

[40] S.M. Ikhdair, R. Sever, Int. J. Mod. Phys. C 20 , 361 (2009).

[41] S.M. Ikhdair, R. Sever, Int. J. Mod. Phys. C 19 , 1425 (2008)

[42] S.M. Ikhdair, M. Hamzavi, Physica B 407, 4198 (2012).

[43] S.M. Ikhdair, M. Hamzavi, A.R. Pazouki, A.H. Behrouz, M. Amirfakhrian, Rom. Rep. Phys. 66, 621 (2014).

[44] G. Chen, Phys. Lett. A 326, 55 (2004).

[45] A. Arda, R. Sever, J. Math. Chem. 50, 971 (2012).

[46] M.R. Spiegel, Schaum's Outline of Theory and Problems of Laplace Transforms, Schaum Publ. Co., New York 1965.

[47] W. Grenier, Relativistic Quantum Mechanics, 3rd ed., Springer, Berlin 2000.

[48] L.J. Slater, Confluent Hypergeometric Functions, Cambridge University Press, London 1960. 